\title{
Defecation and ambulation in a novel environment as determinants of dominance-subordination polarity in the rat
}

GILBERT BECKER, UNIVERSITY OF MANITOBA, Winnipeg, Canada, AND IRBY J. GAUDET, AUBURN UNIVERSITY, Auburn, Alabama

High and low emotional defecators in cross-classification with high and low emotional ambulators were paired with members of their own group and permitted to compete for food. Polarity scores, defined as the winner's proportion-of-time-at-thefood-container scores minus 50, followed a pattern similar to that reported in early-handled and nonhandled rats, but only where defecation was the basis for statistical manipulation.

The authors have recently reported that rats systematically handled in infancy tend to show greater dominance-subordination polarity (DSP) in a food-competition situation than do nonhandled controls (Becker \& Gaudet, 1968). Two of the effects of early handling are an increase in ambulation and a decrease in defecation after placement in a novel environment (Levine \& Broadhurst, 1963). Rats vary, without experimental prompting, in their tendency to "freeze" and to evacuate in a strange environment (Hall, 1934; 1936), and it seems reasonable to suppose that rats naturally high in ambulation and/or low in defecation might show greater DSP, as do early-handled rats. The present study sought to extend the authors' previous findings based on stimulus-defined intervening processes to those based on response-defined ones.

Subjects. The Ss were 24 male rats of Sprague-Dawley derivation. They were selected from a larger group on the basis of bolus and ambulation scores taken in a 2-min open-field test. Scores on each variable were classified as high or low, and four groups of six rats each were formed accordingly: the high bolus-high ambulation group (HH), the low bolus-low ambulation group (LL), the high bolus-low ambulation group (HL), and the low bolus-high ambulation group (LH). All 12 low-bolus Ss dropped no boli during the test. The mean number of boli dropped among the 12 high-bolus Ss was 2.75. The mean number of squares traversed among the 12 low-ambulation Ss was 7.25, and among the 12 high-ambulation Ss, 43.50.

Apparatus. The box used in the open-field test had a 40 -in.-sq base and 18 in. walls. On the floor $8 \times 8$ in. squares were marked off in white lines in an otherwise all black interior. A $300-W$ bulb extended 29 in. above the floor from an aluminum-foil-covered pyramidal apex. Auditory stimulation was not used.

The apparatus used for testing DSP has been described in detail and illustrated elsewhere (Becker, 1965). Briefly, it is a rectangular wooden box with a removable Plexiglas top. From each end extends a platform on which a home cage can be secured on its side. When the guillotine door at either end of the box is raised, the rat in the cage can approach the contents of the plastic cylindrical container screwed to the center of the floor.

Procedure. The Ss were habituated to the dominance-test box over a 10-day period, during which an 8-g-per-day feeding schedule was maintained. All Ss by the end of the period had reached the criterion of a .05 min latency in reaching the wet mash in the food container with the exception of one $S$ whose latency was $.12 \mathrm{~min}$. During habituation one $\mathrm{S}$ in the $\mathrm{LH}$ group was found dead, leaving only five Ss in that group to compete.

Competitions began the day after habituation trials were concluded, at which time the food-deprivation schedule was increased to $12 \mathrm{~g}$ per day. The Ss at this stage were approximately 135 days of age. Each $S$ was paired with every other $S$ in his group until a complete round-robin (RR) had been run. The paired Ss were allowed to compete for food over a 5-min period or trial. No $S$ competed more than once a day and a complete $R R$ ran its course over five daily sessions or sets of trials (or over four sessions in the LH group).

On a given competition two cages (with $S$ inside) were placed one on each cage platform. The two doors were raised simultaneously. Each $S$ could then approach the food containet and compete for the wet mash available to only one $S$ at a time because of the small diameter of the container.

Data Treatment. The accumulated-time scores were converted into percentages indicating the amount of time $S$ spent eating at the container relative to the total amount of time both Ss spent eating at the container. The DSP score obtained in a given encounter for both contestants was defined as the winner's percentage score minus 50 . The mean DSP score for each $S$ over a given RR was the observational basis for a 2 by 2 by 3 (bolus by ambulation by $R R$ ) mixed factorial analysis of variance.

Results. Central tendencies are summarized in Fig. 1. The bolus by ambulation interaction effect is marginally significant $(F=4.00$, $\mathrm{df}=1 / 19, \mathrm{p}<.10)$ and the bolus by $\mathrm{RR}$ interaction effect is significant $(F=5.05, d f=2 / 38, p<.02)$. Separate bolus by ambulation factorial analyses for RR1 and RR3 were computed. The interaction effect in $R R 1$ is highly significant $(F=8.30$, $\mathrm{df}=1 / 19, \mathrm{p}<.01$ ), but in RR3 is nil. This contrast accounts for the marginally significant interaction effect in the 3-factor analysis. DSP differences in the $L L$ and HL groups in RR1 are highly reliable $(t=3.43, \mathrm{df}=10, \mathrm{p}<.01)$.

The bolus effect in both RR1 and RR3 is marginally significant $(F=3.80, d f=1 / 19, p<.10$ and $F=3.97, d f=1 / 19, p<.10)$ but the direction of difference is not the same. DSP scores in the two low-bolus groups combined are significantly different in RR1 and RR3 $(A=.183, \mathrm{df}=10, \mathrm{p}<.02)$.

Dispersion of DSP scores across groups and RRs is shown in Table 1. The overall variance differences among the four groups are not significant $(C=.446,4 / 5, p>.05)$ given the small number of df. There is an overall variance increase across RRs which is significant at least at the .05 level in view of the use of a test for independent variances $(C=.563,3 / 22, p<.05)$. The overall variances in $R R 1$ and $R R 3$ are highly reliably different $(t=2.91$, $\mathrm{df}=21, \mathrm{p}<.01$ ).

Discussion. With respect to defecation, the present findings are fundamentally similar to those found in the authors' early-handling study. There is the same direction of difference found by the final block of trials (RR3), although the magnitude of difference is not as great. The smaller difference results from failure of the high-bolus groups to decrease in DSP over trials, which in turn may be due to the high-bolus groups' status being somewhat misrepresented; a better label (in view of their mean bolus score of 2.75) might have been had by substituting the adjective medium for high.

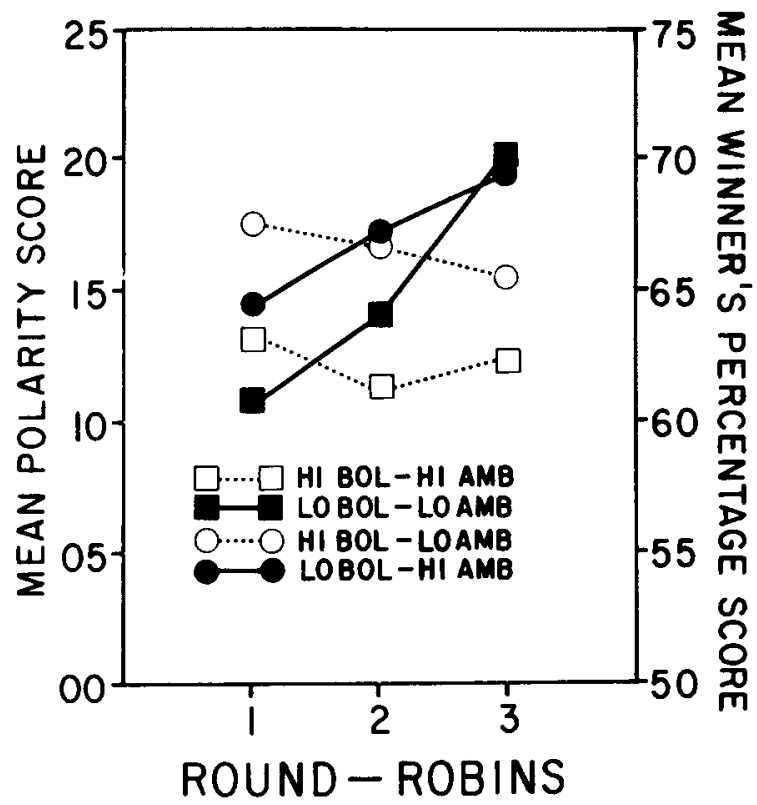

Fig. 1. Polarity score as a function of round-robin. 
Table 1

Standard Deviations of Polarity Scores

\begin{tabular}{lcccc} 
& \multicolumn{4}{c}{ Round-Robin } \\
\cline { 2 - 5 } Group & 1 & 2 & 3 & Total \\
\hline HH & 3.66 & 3.52 & 5.82 & 4.26 \\
LL & 2.56 & 4.96 & 9.82 & 7.28 \\
HL & 4.14 & 6.29 & 6.20 & 5.39 \\
LH & 2.76 & 3.64 & 5.43 & 4.30 \\
\hline Total & 4.05 & 5.06 & 7.36 & 5.69 \\
\hline
\end{tabular}

The initial reversal of direction of difference in the low- and high-bolus groups was not found in the authors' previous study and is attributable solely to the low-ambulation Ss. The reason for this is not clear. Perhaps Ss in the previous study tended to "freeze" less, and if so, the tendency may have resulted from the fact that they did not have to meet a novel opponent on succeeding trials as did the present Ss (Becker \& Flaherty, 1966).

The null findings with respect to ambulation are not too surprising in view of a recent report on the lack of consistency of ambulation scores across open-field trials (Whimbey \& Denenberg, 1967). One implication is that in looking for response-defined criteria for selecting Ss for properties similar to those achieved through early handling, one should give priority to emotional defecation and discard "freezing" behavior (except, perhaps, under highly controlled and understood conditions of testing).

The failure to find variance differences among the groups seems at first to contrast with the obvious variance differences reported between early-handled and nonhandled rats in the previous study. The explanations offered for that difference were in terms of differential susceptibility to experimental treatment, and of nonhandled Ss' DSP scores being closer to one end (bottom) of the scale. The present results are comprehensible when it is realized that "nature" has selected out those who were insensitive to her "manipulation"; further, because the "medium-bolus" groups did not decrease in DSP to the level reached by the nonhandled controls in the previous study, they could not be considered much closer to one end of the scale.

Increases in variability over trials also seem to contrast with findings in the previous study, which indicated first an increase and then a return to the initial level of variability. One may speculate that the curvilinear trend reflects a consolidation process that is common to groups of all sizes, but takes longer to run its course in larger groups, as does the consolidation of dominance-hierarchy stability (Becker \& Flaherty, in press). Accordingly, one would predict, had additional RRs taken place, that variability would have returned to the level seen in RR1.

\section{REFERENCES}

BECKER, G. Social dominance and subordination in the rat as a function of post weaning electrical stimulation. J. genet. Psychol, 1965, 107, 349-369.

BECKER, G., \& FLAHERTY, T. B. Effects of postweaning tactual stimulation on emotionality and social dominance in the rat. Psychol Rep., 1966, 19, 363-366.

BECKER, G., \& FLAHERTY, T. B. Group size as a determinant of dominance-hierarchy stability in the rat. J. comp. physiol. Psychol, in press.

BECKER, G., \& GAUDET, l. J. Dominance-subordination polarity in early-handled and nonhandled rats. Psychon. Sci, 1968, 11, 115-116.

HALL, C. S. Emotional behavior in the rat: I. Defecation and urination as measures of individual differences in emotionality. J. comp. Psychol, $1934,18,385-403$.

HALL, C. S. Emotional behavior in the rat: III. The relationship between emotionality and ambulatory activity. J. comp. Psychol, 1936, 22, 345-352.

LEVINE, S., \& BROADHURST, P. L. Genetic and ontogenetic determinants of adult behavior in the rat. J. comp. physiol. Psychol, 1963, 56, 423-428.

WHIMBEY, A. E., \& DENENBERG, V. H. Two independent behavioral dimensions in open-field performance. J. comp. physiol Psychoh, 1967, $63,500-504$. 